\title{
Compression or obstruction: Prospective analysis of the function of the adjustable transobturator male system (ATOMS) based on pre- and postoperative urodynamic data
}

Fabian Queissert ${ }^{1}$; Benedict Bruecher ${ }^{1}$; Sonja Ruiz ${ }^{2}$; Miguel Virseda-Chamorro ${ }^{3}$; Andres J. Schrader ${ }^{1}$; Javier C. Angulo ${ }^{2}$

${ }^{1}$ University Hospital of Muenster, Department of Urology and Pediatric Urology, Muenster, Germany; ${ }^{2}$ Clinical Department, Universidad Europea de Madrid; Hospital Universitario de Getafe, Getafe, Spain

Cite as: Queissert F, Bruecher B, Ruiz S, et al. Compression or obstruction: Prospective analysis of the function of the adjustable transobturator male system (ATOMS) based on pre- and postoperative urodynamic data. Can Urol Assoc J 2021 December 21; Epub ahead of print. http://dx.doi.org/10.5489/cuaj.7551

Published online December 21, 2021

Corresponding author: Dr. Fabian Queissert, University Hospital of Muenster, Department of Urology and Pediatric Urology, Muenster, Germany; Fabian.Queissert@,ukmuenster.de

$* * *$

\section{Abstract}

Introduction: This analysis, based on pre- and postoperative urodynamic data, is the first to elucidate the influence of the adjustable transobturator male system (ATOMS, A.M.I. GmbH, Feldkirch, Austria) on the lower urinary tract and disclose possible obstructive properties.

Methods: A prospective study was performed in patients who had stress urinary incontinence and were scheduled for ATOMS implantation after radical prostatectomy. Apart from continence assessment (24-hour pad test, International Consultation on Incontinence Questionnaire-Urinary Incontinence Short Form [ICIQ-SF]), urodynamic testing was done with International Continence Society (ICS)-standardized pressure-flow analysis before and after ATOMS implantation/adjustment. The Wilcoxon signed-rank test was used for statistical analysis.

Results: The analysis included 12 consecutive patients from two centers (mean 69 years) with a mean followup of 246 days. Median urine leakage dropped from 240 (72-1250) to 70 (0-700) $\mathrm{g} / 24$ hours postoperatively, with a pad reduction of 4 to 0.9 pads/day. Pressure-flow analysis revealed a significant change only in the bladder outlet obstruction index (BOOI). The bladder contractility index, intravesical pressure conditions, and uroflowmetry were not significantly affected. None of the patients showed de novo obstruction postoperatively in the ICS analysis. Conclusions: The ATOMS significantly increases the BOOI in conjunction with good continence results. However, no case reached pathological level according to the BOOI and thus there is no potential danger to the lower urinary tract or urethral integrity. 


\section{Introduction}

Various surgical options are available for treating male stress incontinence after radical prostatectomy. Apart from the artificial urinary sphincter (AUS) as the gold standard, fixed slings have become established as well as adjustable urethral compression systems like the Argus, Reemex, ProAct and more recently the adjustable transobturator male system (ATOMS). Although a short follow-up and comparatively low success rates are criticized in the guidelines for the ATOMS (1), a current review examining a longer follow-up and including the new generation ATOMS with a silicone-covered scrotal port (SSP) demonstrates higher success rates with continued low complication rates (2). However, little is known as yet about the mechanism of action of the ATOMS, its safety, and its influence on lower urinary tract function. As a compression device, the ATOMS is implanted under the bulbar urethra, where obstruction is at least conceivable, depending on the filling level. This could exert a negative influence on bladder integrity and lead to urethral damage in the follow-up. After correct retrobulbar implantation of the fixed sling Advance (Boston Scientific), urodynamic testing revealed no obstructive component and no relevant influence on voiding (3). Similarly, this prospective noninterventional feasibility study in a small series of patients from 2 centers is the first to urodynamically evaluate ATOMS implantation for its effects on bladder function and to discard a possible obstructive implant property.

\section{Methods}

The opportunity to participate was offered to men who suffered from stress urinary incontinence $>1.5$ years after radical prostatectomy and were scheduled for ATOMS implantation during the investigation period between 11/2019 and 12/2020. Approval by the Ethics Committee of Westphalia-Lippe was obtained before starting the study (2019-248-f-S). Patients with previous incontinence surgery, active urethral/bladder-neck stenosis or former surgical treatment of any source of bladder outlet obstruction were excluded. Cystourethroscopy verified eligibility for ATOMS implantation by assessing residual sphincter function and excluding obstruction. Guideline-based urodynamic testing was done to check the indication and exclude relevant urinary stress incontinence (4). It was ICS-standardized (International Continence Society) and regularly took place in a standing position (5). Apart from cystometric data acquisition (bladder volume at first and strong desire, detrusor overactivity, maximum detrusor pressure, and compliance), a pressure-flow study was performed to record maximum uroflow, residual urine, bladder contractility index $\left(\mathrm{BCI}=\mathrm{P}_{\text {Det.Qmax }}\right.$ (Detrusor-Pressure at maximum flow) $+5 \mathrm{x} \mathrm{Q}_{\max }$ (maximum flow) $)$ and bladder outlet obstruction index $\left(\mathrm{BOOI}=\mathrm{P}_{\operatorname{Det}} \mathrm{Q}_{\max }-2 \mathrm{x} \mathrm{Q}_{\max }\right)$. The latter is regarded as a valid parameter for assessing infravesical obstruction and enables classification of micturition as unobstructed $(\mathrm{BOOI}<20)$, equivocal (BOOI 20-40) or obstructed (BOOI $>40)$ according to ICS criteria (6).

After screening and consent, preoperative continence data were recorded (pads/24 h; 24-h pad test; International Consultation on Incontinence Questionnaire - Short Form (ICIQ-SF)). All patients underwent perineal ATOMS SSP implantation using the method described by Seweryn et al (7). The intervention was performed at both participating centers by a surgeon with 
appropriate expertise (> 70 ATOMS). The system was initially filled with isotonic saline solution after venting by passive pressure equalization. Adjustment was done until either satisfactory continence was achieved or discomfort prevented further filling of the system. Urodynamic and continence parameters were again recorded after completing adjustment but 3 months after the intervention at the earliest and 12 months thereafter at the latest. Statistical analysis was done with SPSS for Windows (Version 27.0). The Wilcoxon signed rank test was used to detect any differences in urodynamic parameters. P-values $<0.05$ were considered statistically significant.

\section{Results}

The study included a total of 15 patients who underwent urodynamic testing and subsequent ATOMS implantation in Münster or Getafe between November 2019 and January 2021. Patients had a mean age of 69 years (range 64-72) at the time of surgery and a mean BMI (body mass index) of $26.4 \mathrm{~kg} / \mathrm{m}^{2}$. During follow-up, two subjectively satisfied patients refused postoperative urodynamic testing, and in another case catheter insertion proved difficult and was prematurely terminated at the patient's request. A median of 1.0 (range 0-3) adjustment was made in the 12 remaining patients, and the device had a median filling volume of $12.0 \mathrm{ml}$ (range 6.5-19.0). Median urine leakage dropped from $240.0 \mathrm{~g} / 24 \mathrm{~h}$ (range 72-1250) preoperatively to $70 \mathrm{~g} / 24 \mathrm{~h}$ (range $0-700$ ) postoperatively (Fig. 1) with a pad reduction from a median of 4 pads/24h to 0.9 pads $/ 24 \mathrm{~h}$. Nine patients (75\%) achieved social continence ( $0-1 \mathrm{pad} / 24 \mathrm{~h})$ with mean urine leakage of $3 \mathrm{~g} / 24 \mathrm{~h}$. The median ICIQ-SF sum score dropped from 16.0 preoperatively to 5.5 postoperatively (ICIQ-SF 1: $4.0 \rightarrow 1.0$; ICIQ-SF 2: $4.0 \rightarrow 2.0$; ICIQ-SF 3: $7.5 \rightarrow 2.5$ ). The median postoperative PGI-I (Patient Global Impression of Improvement) was 2.0 ( $1=$ very much better, 2 $=$ much better; $3=$ a little better; $4=$ no change; $5=$ a little worse; $6=$ much worse; $7=$ very much worse).

\section{Urodynamics - Cystometry}

Cystometric results are presented in Table 1. There were no significant differences between the following pre- and postoperative cystometric parameters: bladder volume at 1 st and strong desire, maximum detrusor pressure, and compliance. Two patients had de novo detrusor overactivity (DO) postoperatively. In another case DO was present initially and no longer detectable postoperatively. In both cases with DO, postoperative volume of $1^{\text {st }} \mathrm{DO}$ was higher than the maximum cystometric bladder volume in preoperative urodynamic.

\section{Urodynamics - Pressure flow study}

Maximum uroflow, residual urine and BCI (Fig. 2) did not change significantly in the Wilcoxon signed rank test. Median maximum detrusor pressure $\left(25 \rightarrow 32 \mathrm{cmH}^{2} \mathrm{O}(\mathrm{p}=0.08)\right)$ and maximum intravesical pressure $\left(59 \rightarrow 75 \mathrm{cmH}^{2} \mathrm{O}(\mathrm{p}=0.09)\right)$ tended to be higher postoperatively. The BOOI was the only parameter to show a significant increase from -14 preoperatively to 5.5 postoperatively $(\mathrm{p}=0.034)$. One case $(8.3 \%)$ was classed higher postoperatively according to ICS criteria (changed from unobstructed to equivocal, Fig. 3). After surgery and adjustments, none of the patients had obstructed micturition according to ICS criteria. 


\section{Discussion}

Eight years after the device reached market maturity, our study undertook the first urodynamic analysis of bladder storage and emptying function before and after ATOMS implantation. Direct ventral compression of the bulbar urethra by the silicone cushion significantly increased the BOOI, but de novo obstruction did not reach a pathologic level according to the BOOI in all examined cases. The absence of obstruction could be due to the urethra gaining many degrees of freedom through compression on only one side. Thus, the ATOMS appears to function not just by statically obstructing the urethra but rather by indirectly supporting the external sphincter muscle. In their study on fixed sling function, Rehder et al (8) already discussed the relevant function of the urethral bulb to maintain continence. Physical activity causes physiological contraction of the bulbospongiosus muscle, which increases pressure in the bulb of corpus spongiosum and consecutively leads to additional narrowing of the urethral lumen in the distal part of the rhabdosphincter. This principle may be assumed for the ATOMS, especially since the implantation technique propagated by Seweryn et al (7) leaves the bulbospongiosus muscle intact and does not require exposure of the urethra. Therefore, in our opinion, the mechanism of action of the ATOMS involves not only direct compression of the proximal penile and bulbar urethra but also an indirect effect through increased pressure in the corpus spongiosum and consecutive narrowing of the membranous urethral lumen. In line with our study, no obstructive effect or relevant voiding dysfunction was detected for the fixed transobturator retrobulbar sling (Boston Scientific) (3). For the adjustable ProAct system, on the other hand, Utomo et al (9) demonstrated an obstructive component with a significant increase in the BOOI from -7.4 to 23.1 (i.e.; equivocal, according to ICS criteria) in most of the patients treated. This could be due to two-sided contralateral compression of the urethra. Despite long availability of these devices, thus far there are no publications on urodynamic analyses before/after implantation of an AUS or any of the other adjustable continence systems.

To predict possible consequences of iatrogenic obstruction, it is helpful to consider the long-term consequences of obstruction assessed by Thomas et al (10). No complete deterioration of urodynamic pressure-flow parameters was seen in the 10-year follow-up of patients with bladder outlet obstruction (10). Detailed analysis, however, revealed significantly more prevalent DO, reduced detrusor contractility and increased residual urine in the group that did not undergo deobstruction during the entire observation period.

Another possible and more direct consequence of long-term obstruction is atrophy of the compressed tissue with the risk of urethral erosion. In the first publication on ATOMS, Seweryn et al (7) already noted that absence of the circular compression as that created by the AUS is associated with a markedly lower urethral erosion rate. Even larger long-term follow-up studies revealed no relevant prevalence of urethral erosion after ATOMS implantation (11). In contrast, Utomo et al already detected unspecified tissue damage requiring revision during a short followup in $3.7 \%$ of patients who received the adjustable balloon device ProAct (9). Urethral erosion after AUS implantation is also attributed to urethral atrophy caused by circular compression and impaired perfusion $(12,13)$. Apart from the risk of urethral erosion, urethral atrophy in the cuff area also harbors the risk of recurrent incontinence through inadequate urethral closure. Our 
analysis therefore supports the view that, unlike the AUS, the ATOMS carries no risk of relevant urethral atrophy and consecutive erosion. The possibility of adjustment via the scrotal port during follow-up additionally reduces the risk of recurrent incontinence as a reason for revision/reimplantation.

Cystometry showed no significant changes in our cohort. Low compliance was not observed during our short follow-up. Two patients in the group we examined had de novo DO, though this did not influence the subjective outcome or continence results. The fact, that preoperative maximum cystometric volume in both cases were lower than volume at $1^{\text {st }} \mathrm{DO}$ in postoperative surgery let us assume, that preoperative DO could be underestimated due to early urine leakage. Schoenburg et al (14) found no de novo DO in their prospective study in 361 patients with an ATOMS. Open to debate is the fact that urodynamic testing was not done regularly in the preoperative phase and that the postoperative symptoms of an overactive bladder were initially recorded only via questionnaires. Only 18 of 361 patients underwent urodynamic testing, which revealed no DO and only one case of low compliance. Thus, DO could have remained undetected in asymptomatic/satisfied patients.

\section{Limitations}

The BOOI has been originally developed to estimate the degree of obstruction in men with an enlarged prostate. It has to be annotated that it is not been validated for use after radical prostatectomy. Due to its widespread use, the well investigated connection between higher BOOI and bladder deterioration and the fact that other working groups also applied the BOOI $(3,9)$, we decided to use it in our analysis. The basically short follow-up harbors the risk of underestimating effects of the ATOMS, since any subsequent adjustments could cause more severe obstruction. Moreover, influences on bladder function often become apparent only in the long term (10). However, comparison of the ATOMS filling volume in our study (median $12 \mathrm{ml}$ ) with the results of the long-term follow-up in the Iberian study (11) discloses only a marginal difference in the adjusted volume (mean $13.2 \mathrm{ml}$ ). Furthermore, our patient with the highest filling volume $(19 \mathrm{ml})$ still had a BOOI of only -3 . It therefore seems to us that our results have sufficient informative value.

\section{Conclusions}

The ATOMS is a safe and successful treatment method. Despite a slight increase in the BOOI, none of the patients treated show any indication of relevant obstruction according to BOOI or deterioration of bladder function. Long-term urethral integrity and bladder function may be assumed but require a longer follow-up in a larger number of patients.

Disclosures: Dr. Queissert and Dr. Angulo have lectured and been consultants for AMI GmbH. There remaining authors report no personal or financial conflicts. 


\section{References}

1. Burkhard F.C. BJLHR, Cruz F., Lemack G.E., Nambiar A.K., Thiruchelvam N., Tubaro A. EAU Guidelines on urinary incontinence Edn. presented at the EAU Annual Congress Amsterdam 20202020 [Available from: https://uroweb.org/guideline/urinaryincontinence.

2. Esquinas C, Angulo JC. Effectiveness of Adjustable Transobturator Male System (ATOMS) to Treat Male Stress Incontinence: A Systematic Review and Meta-Analysis. Adv Ther. 2019;36(2):426-41.

3. Soljanik I, Becker AJ, Stief CG, Gozzi C, Bauer RM. Urodynamic parameters after retrourethral transobturator male sling and their influence on outcome. Urology. 2011;78(3):708-12.

4. Abrams PC, L.; Khoury, S.; Wein, A. Incontinence 5th Edition 2013. Paris: EAU-ICUD; 2013.

5. Schafer W, Abrams P, Liao L, Mattiasson A, Pesce F, Spangberg A, et al. Good urodynamic practices: uroflowmetry, filling cystometry, and pressure-flow studies. Neurourol Urodyn. 2002;21(3):261-74.

6. Griffiths D, Hofner K, van Mastrigt R, Rollema HJ, Spangberg A, Gleason D. Standardization of terminology of lower urinary tract function: pressure-flow studies of voiding, urethral resistance, and urethral obstruction. International Continence Society Subcommittee on Standardization of Terminology of Pressure-Flow Studies. Neurourol Urodyn. 1997;16(1):1-18.

7. Seweryn J, Bauer W, Ponholzer A, Schramek P. Initial experience and results with a new adjustable transobturator male system for the treatment of stress urinary incontinence. $\mathrm{J}$ Urol. 2012;187(3):956-61.

8. Rehder P, Staudacher NM, Schachtner J, Berger ME, Schillfahrt F, Hauser V, et al. Hypothesis That Urethral Bulb (Corpus Spongiosum) Plays an Active Role in Male Urinary Continence. Adv Urol. 2016;2016:6054730.

9. Utomo E, Groen J, Vroom IH, van Mastrigt R, Blok BF. Urodynamic effects of volumeadjustable balloons for treatment of postprostatectomy urinary incontinence. Urology. 2013;81(6):1308-14.

10. Thomas AW, Cannon A, Bartlett E, Ellis-Jones J, Abrams P. The natural history of lower urinary tract dysfunction in men: minimum 10-year urodynamic follow-up of untreated bladder outlet obstruction. BJU Int. 2005;96(9):1301-6.

11. Angulo JC, Virseda-Chamorro M, Arance I, Ruiz S, Ojea A, Carballo M, et al. Longterm outcome of adjustable transobturator male system for stress urinary incontinence in the Iberian multicentre study. Neurourol Urodyn. 2020;39(6):1737-45.

12. Queissert F, Huesch T, Kretschmer A, Anding R, Kurosch M, Kirschner-Hermanns R, et al. Artificial Urinary Sphincter Cuff Size Predicts Outcome in Male Patients Treated for Stress Incontinence: Results of a Large Central European Multicenter Cohort Study. Int Neurourol J. 2019;23(3):219-25.

13. Simhan J, Morey AF, Zhao LC, Tausch TJ, Scott JF, Hudak SJ, et al. Decreasing need for artificial urinary sphincter revision surgery by precise cuff sizing in men with spongiosal atrophy. J Urol. 2014;192(3):798-803.

14. Schonburg S, Bauer W, Mohammed N, Brossner C, Fornara P. De novo OAB After ATOMS: An Underestimated Problem or a Rare Side Effect? Front Surg. 2019;6:72. 


\section{Figures and Tables}

Fig. 1. Continence results at time of postoperative urodynamics.

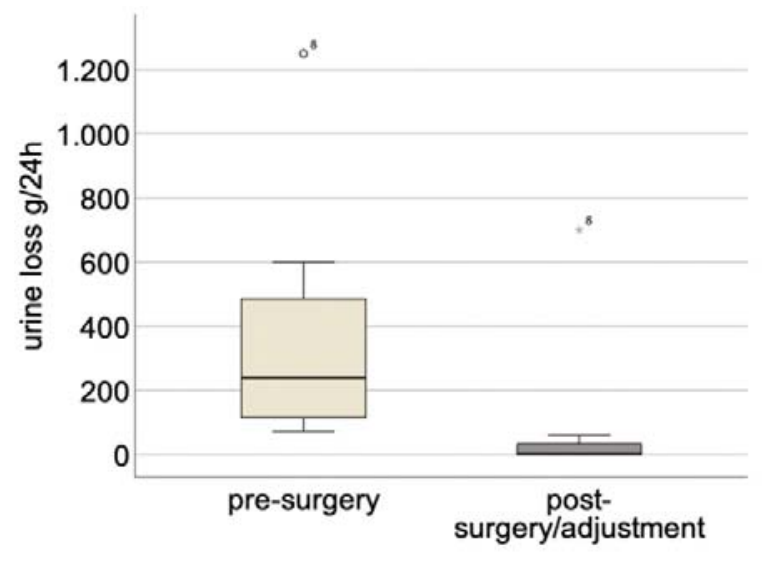

Fig. 2. Changes in Bladder Contractility Index $\left(B C I=P_{\text {Det.Qmax }}+5 x Q_{\max }\right)$.

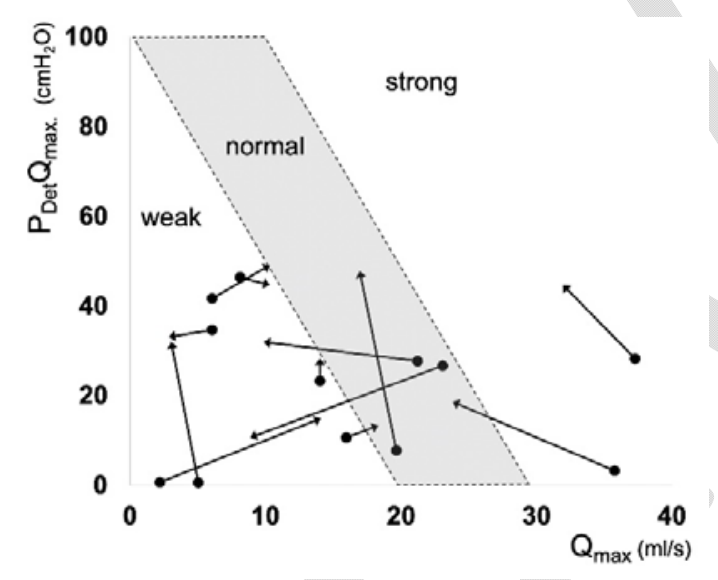


Fig. 3. Changes in Bladder Outlet Obstruction Index (BOOI $\left.=\mathrm{P}_{\operatorname{Det}} \mathrm{Q}_{\max }-2 \mathrm{x} \mathrm{Q}_{\max }\right)$.

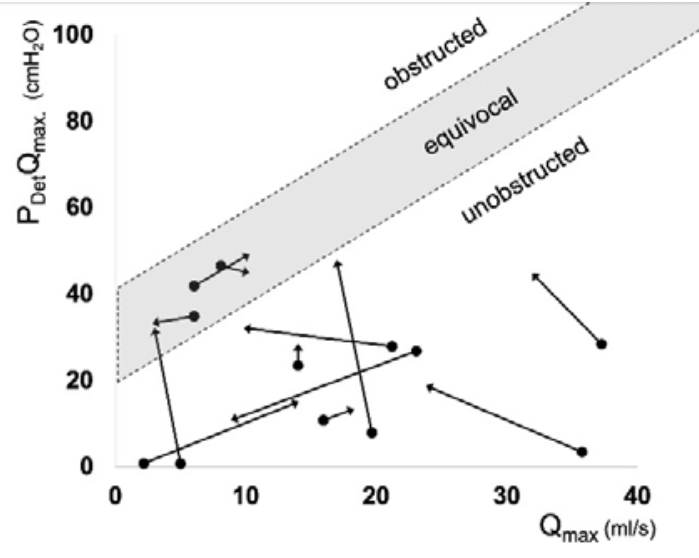

\begin{tabular}{|c|c|c|c|}
\hline Urodynamic parameter & Preoperative & Postoperative & $\begin{array}{c}p(\text { Wilcoxon } \\
\text { signed rank test) }\end{array}$ \\
\hline \multicolumn{4}{|l|}{ Cystometry } \\
\hline Median cystometric bladder capacity, ml (range) & $309(97-569)$ & $359(120-502)$ & 0.88 \\
\hline Median volume first desire, $\mathrm{ml}$ (range) & $181(20-403)$ & $205(119-356)$ & 0.43 \\
\hline Median volume strong desire, $\mathrm{ml}$ (range) & $260(97-491)$ & $309(120-480)$ & 0.75 \\
\hline Detrusor overactivity (yes/no) & $4 / 8$ & $5 / 7$ & 1.0 \\
\hline $\begin{array}{l}\text { Median maximum detrusor pressure, } \mathrm{cmH}_{2} \mathrm{O} \\
\text { (range) }\end{array}$ & $10(2-45)$ & $19(2-52)$ & 0.24 \\
\hline Median detrusor compliance, $\mathrm{cmH}_{2} \mathrm{O} / \mathrm{ml}$ (range) & $55(8-223)$ & $126(15-224)$ & 0.53 \\
\hline \multicolumn{4}{|l|}{ Pressure flow study } \\
\hline Median maximum uroflow $\left(\mathrm{Q}_{\max }\right), \mathrm{ml} / \mathrm{s}$ (range) & $15.0(2-37)$ & $12.0(3-32)$ & 0.23 \\
\hline Median residual urine, $\mathrm{ml}$ & $1.0(0-185)$ & $3.0(0-372)$ & 0.51 \\
\hline Median max detrusor pressure, $\mathrm{cmH}_{2} \mathrm{O}$ (range) & $25(0-46)$ & $32(10-49)$ & 0.08 \\
\hline Median max vesical pressure, $\mathrm{cmH}_{2} \mathrm{O}$ (range) & $59(20-117)$ & $75(25-108)$ & 0.09 \\
\hline $\begin{array}{l}\text { Median BOOI } \\
\text { (range) }\end{array}$ & $-14(-69-30)$ & $5.5(-32-29)$ & $0.034^{*}$ \\
\hline $\begin{array}{l}\text { Median BCI } \\
\text { (range) }\end{array}$ & $89(10-213)$ & $96(40-199)$ & 1.0 \\
\hline
\end{tabular}

${ }^{*}$ Statistically significant. ATOMS: adjustable transobturator male system; BCI: bladder contractility index; BOOI: bladder outlet obstruction index. 